\title{
BEYOND GRAPHS OR CHARTS: VISUALISING WEB STATISTICS WITH NATURAL DISPLAYS IN PHYSICAL SPACE
}

Michael Hohl

CCI / Art and Design Research

University of Hertfordshire

College Lane Campus

Hatfield, Hertfordshire

United-Kingdom

m.hohl@herts.ac.uk

http://sitem.herts.ac.uk/artdes_research/ind.html

\begin{abstract}
Screen-based graphs, charts or diagrams help us to understand how visitors make use of a website. Yet, while we experience the world around us with all our senses this experience is mostly introspect and intellectual. It does not address our other senses, such as hearing or touch and conveys even less of the experience of being visited. It is more visual and informative then sensual or aesthetic. This research investigates ways of "visualising" the current presence of visitors of a website beyond the screen as an experience in physical space in an application of calm technology with a natural display.
\end{abstract}

\section{INTRODUCTION}

"Our objective is not the expression of knowledge or information, but the living world itself. We want to come in contact with other people and the living world, not IT equipment or interface design - they should be nothing more than the medium."

Yoshiaki Nishimura (Nishimura, 1997)

From ubiquitous computing and ambient displays to "non-visual multi-modal visualization" and fun technologies.

In 1991 Marc Weiser wrote "The most profound technologies are those that disappear. They weave themselves into the fabric of everyday life until they are indistinguishable from it." (Weiser, 1991) With this text of Weiser the concept of ubiquitous computing emerged; a world of "intelligent" objects exchanging data with one another and in which this network of objects is superseding a centralised computer network.

Another idea that Weiser introduced was that of information being displayed at the periphery of perception, and thus distinguishing between a centre and a background for digital information. He used artist in residence Natalie Jeremijenko's work "Dangling String" (Weiser, 1995) as an example of what he described as calm technology. The artist had attached an eight foot long plastic string to a small electric motor fixed to the ceiling in a corridor of a Xerox Parc office and connected it to an ethernet cable. By visibly and audibly whirling around at different speeds the string indicated light or heavy ethernet traffic. From this example Weiser concluded that digital information did not necessarily have to be confined to a computer screen but could also include everyday objects, 
describing such an application as "fun and useful." These calm technologies, he wrote, possibly were the "key challenge in technology design for the next decade." His argument was that there was a growing need for calm technologies as a result of information technology more often being the enemy of calm with mobile phones, email, pagers and the web inundating us with information. He observed that the difference lay in the way in which each engaged our attention. His associations of information technologies being "fun and useful" were a prediction for future developments.

Both of Weisers concepts, that of ubiquitous computing and of peripheral perception were later explored by Hiroshi Ishi and The Tangible Media Group at the MIT Media Lab. Here the terms "tangible bits" or tangible computing and ambient media were coined. Ambient media (Ishi et. al, 1998a, 1998b) were seamless interfaces integrating people and digital information through interactive objects. This led to further research into ambient displays. These ambient displays were displays that resided in the background, unobtrusive, not interrupting the attention of humans occupied with a primary task, but were available when needed, similar to a clock upon the wall at the periphery of human perception.

Since then the design of information technologies has developed further. Media theorist Lev Manovich observes in retrospect that instead of the computer becoming invisible, as Weiser predicted, in fact the opposite had taken place. Today, he writes, we were surrounded by interactive devices such as Laptops, iPods, mobile phones and handhelds yet the interaction with them had changed. It was "treated as an event [...] a carefully orchestrated experience." resulting in a "theatrisation" of information technology design (Manovich, 2007).

\section{Non-visual multi-modal visualization}

Another case of this theatralisation relevant to our investigation pertains to the relationship between digital technologies and visualization of data. Software applications permit us to visualise data in entirely new and revolutionary ways between visual representations and other sensory modalities, for example sound to visuals or visuals to sound. A simple application of these are visualiser plug-ins for MP3 players. Here distinct properties of the sound such as pitch and beat are transformed in realtime into animated visuals upon the screen. Often it is possible to choose from among various visualization modes, or "themes," with entirely different visual styles being synchronised live with the playing music. Properties of sound are being transformed into a purely entertaining colourful visual experience beyond informative and utilitarian graphs or charts.

Another example for a "non-visual visualization" with experiential qualities is the installation "Tide"1 created in 2004 by the British artist Luke Jerram. "Tide" transforms the gravitational pull of the moon into an immersive acoustic experience. The installation consists of three large rotating glass spheres which are filled with water, each mounted on a tripod and positioned several metres apart from each other in a spacious gallery setting. A friction device sitting on the rim of each sphere makes it swing in vibrant overtones resonating within the space. The gravitational pull created by the celestial bodies, mainly by the moon, is measured by a gravity meter located in the gallery and streamed to a 
computer. This computer adjusts the water levels within the glass spheres synchronous to the live data stream thus determining its sound pitch. As the gravitational forces change over time the water levels within the glass spheres rise or sink accordingly, changing the sound of each glass in a live synchronisation with the actual movement of the celestial bodies sun, earth and moon; the resulting resonating room-filling chorus brings to mind the sound of singing wine glasses, yet larger and more visceral. "Tide" can be seen as a scientific instrument in a classical tradition; on one hand it is a comprehensive technical instrument that measures, yet on the other it is a sensual, aesthetic and poetic experience. It employs visualization across sensory modalities or "transformation of data" from one medium - gravity - to another - sound - to allow us to experience changes of a natural force we are continuously immersed in yet which to detect are beyond the capacity of our limited human senses. Technology, transformation, telematics and the physical world come together in this piece forming a poetic whole.

Such an extended concept of data visualization may have been on the mind of Lev Manovich when he wrote his 2001 essay "The Anti-Sublime Ideal in New Media" (Manovich, 2001). In this text he describes "mapping data" as "one of the most fruitful research directions in new media art." But why has this mapping of data received more attention from the arts then from information design? Additionally most examples of visualization-art still mostly take place upon the computer screen. With "Tide" we have seen an example of an experiential quality beyond the screen that combines useful aspects in combination with aesthetic ones - this is the area our research focusses upon.

\section{Considerations for a display indicating the presence of a visitor to a website}

Our research focusses upon affordances for experiential ambient displays that allow the author or owner of a website to become aware of a visitor to this site. This display should be located beyond the computer screen in physical space. How do author and visitor become aware of each other with current technologies?

Basically there are three approaches: Direct and indirect textual communication, analytical statistic tools and web-based textual presence indication. With the rise of Web 2.0 the connection between web-authors and their readers has become much closer. The tools facilitating this development include comment-functions on almost every web-page, email and also social bookmarking sites such as Delicious or Reddit. All of these require direct or indirect textual communication and time-consuming active participation. They involve certain degrees of intrusiveness for the recipient as well. Besides their usefulness it appears that they are far from being calm technologies in Marc Weiser's sense. They demand action, involve and can overwhelm the user.

Beyond direct communication with visitors an author has access to analytical web-based tools which provide statistical information about his audience. Among others these include from which city or country visits originate from, which links from other sites visitors have been following or which search phrases they have been using to find the website. Worth noting in this context is that while the voice of the author's writing and the communication among author and visitor may be personal, these statistics are not. Usually the information is anonymised providing no reference for individual visitors interests. Additionally the statistical data is generated once a day only and examined asynchronously after it has been generated. A third and passive way of indicating presence and an awareness of visitors is 
practised by some websites. This information consists of the textual display of the number of guests and members currently present on the site (Figure 1). This type of presence indication still is the exception and not the rule and although it has a vivid emotional component is more of an informative resource.

\section{Technological and conceptual approach}

Recently though there have been various attempts, especially by authors with software programming skills, to remedy this situation by creating physical web-visit indicators beyond the computer screen. Dimitrios Vlastaras has created such an indicator with an Arduino ${ }^{2}$ microcontroller. He writes "[...] ]every time somebody visits my website, a LED light blinks once in my room, and I get a warm feeling of a visitor."(Vlastaras, 2008) Alexander Weber, another owner of a website temporarily connected a physical bell to his server which rang with every visit. He writes: "Literally. It puts a smile on your face, every time someone hits your blog. "(Weber, 2007) Referring to its intrusive properties he adds: "And it is a great way to annoy your colleagues or your girl friend." These two examples show that there is a strong interest in creating an awareness of a visitor instantaneously and also to "visualise" this presence beyond the screen as an aesthetic and emotional experience as opposed to an intellectual one. Yet, what are the conceptual approaches underlying these display ideas? What is the relationship between the visit - and its visualization? How do we know if the conceptual mapping or metaphor is appropriate? While Dimitrios' LED is rather abstract and ambient in that it draws little attention, Alexander's sounding bell reminds of a real visitor ringing at the door. What actually is this virtual event of a visit to a website?

We believe that experiential displays - emotional experiences - such as these will provide a beneficial link between authors of websites and their audiences. These displays transform data beyond statistical visualizations and verbose communication by creating an awareness of the presence of a visitor in real-time. One aim of our research is to create this sense of connectedness without being overly intrusive. Another aim is to explore the aesthetic side of this experience in that it should be located beyond the screen in physical space and be perceived as "natural." Natural in this sense refers to a combination of background activities such as a breeze of air or the sound of trickling water. Both easily embed themselves into a working background without being perceived as too artificial.

What do we want about the visitors of our website? Which information is interesting? Which is relevant? What are the constraints involved? As we have seen before the rich geographical and contextual data created by analytical tools is not as easily available in real-time while it is live information that we are interested in. Live information about visitors can be retrieved for example by websites that are generated out of a database. The content of these websites is stored in a database and generated upon request from a browser. This system automatically registers the unique IP address and the time that a visit, or session, began and it is relatively simple to retrieve this data. As a result the initial information available for display is the time a visitor arrives at the site and his or her unique

2 Arduino is a microcontroller built by a community of developers as an open-source project. Available at http://www.arduino.cc/ accessed May 15th 2008. 
IP or Internet Protocol address.

We are in the process of developing an installation piece that is activity specific in that it resembles to a certain degree an actual visit to the main space from which the website is updated. In its current rapid-developmental stage it consists of a screen-based interface (Figure 1) to communicate with and control the microcontroller and an LED that lights up for two seconds every time a new visitor arrives at the website (Figure 2). In its following iteration it will consist of a curtain which will be set in motion by a "silent" fan. The motion of the curtain evokes the idea of an actual visitor opening the door of the room and creating a temporary draft; thus representing a visitor in physical space. A poetic notion that connects the virtual visit to a physical one.

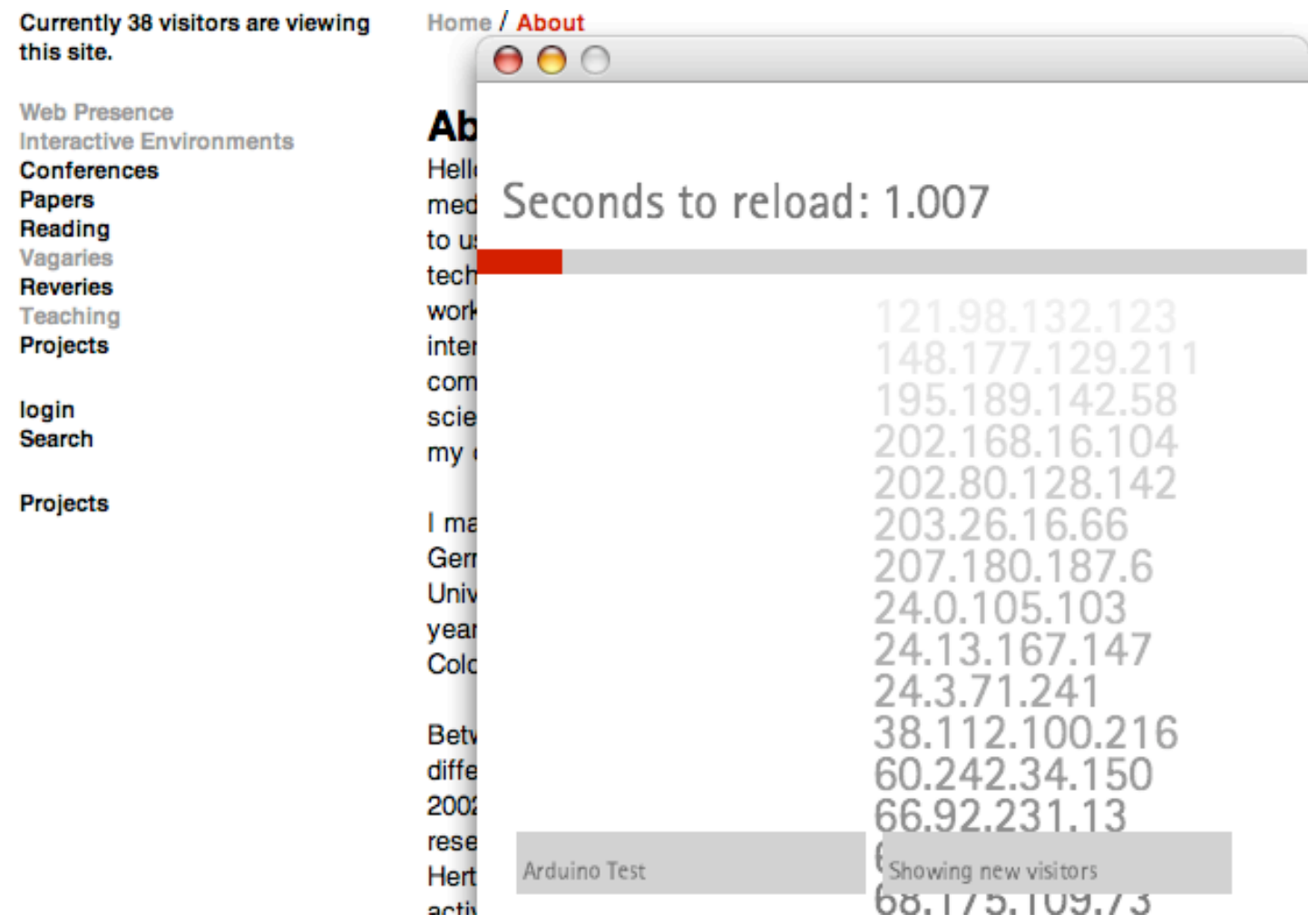

Figure 1. In the background the webpage indicating "Currently 38 visitors are viewing this site," in the foreground the user-interface triggering the external microcontroller, displaying the IP-addresses of all visitors.

We also want to explore how the ambiguity of the medium is perceived. A regular draft could also cause the curtain to move and the individual has to trust his senses. Redundancy of ambient visuals and sound will also play a crucial role in our investigation. As we use a "silent" fan to animate the curtain an additional sound will accompany the motion, reinforcing the perceived signal.

Among our initial research questions are: How much transparency is useful? How much opacity is necessary? Which indicated states are meaningful for recipients? What is the balance between an intrusive display and ambient qualities? What are the most transparent 
mappings for conditions and actions? How direct do these mappings have to be, to be perceived as meaningful?

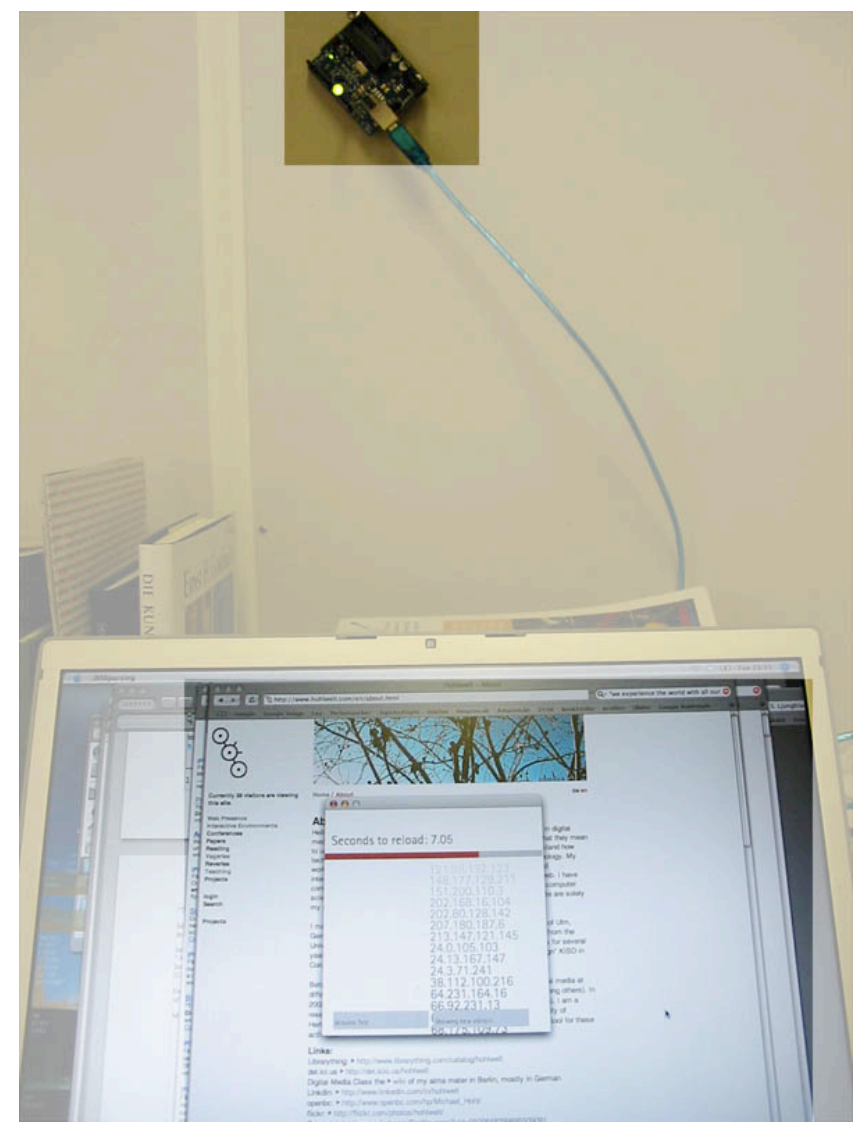

Figure 2: On the screen (pictured below) we simulate a visitor by accessing the website http://www.hohlwelt.com/en. At the same moment the LED (above) lights up for two seconds, indicating this visitor. In its next iteration the LED will be replaced by physical actuators.

\section{CONCLUSION}

Above we have seen that through media conversion the Internet, mobile media, locative media and social data are merging, leaving the screen behind and extending beyond the screen into physical space, into peoples lives. Additionally information technologies have not become invisible but have been transformed into carefully designed emotional experiences. Since the rise of Web 2.0 platforms such as Twitter, Flickr, Dopplr or Delicious in combination with open APIs have provided easy access and a multitude of sources for Mash-ups of different types which can be "visualised" in a multitude of informative as well as experiential ways.

Our "visualization" is not a substitute for the analysis of daily web-statistics but instead pertains to a completely different, the experiential area beyond the screen. It creates a connection between the individuals maintaining the website and the anonymous remote person that visits its "virtual" embodiment: its website - yet passively and beyond the intrusiveness of synchronous communication. This experience is expected to create a closer 
and immediate connection between the individuals updating and administrating the website, a more pleasurable and rewarding web-authoring experience. By exploring how individuals perceive our "displays" we hope to get a better understanding of their affordances and constraints.

Yet there are a range of questions to be considered. Is it desirable at all to aspire for a balance between informative density and an aesthetic, embodied and sensual experience? That may depend on the kind of data involved as well as the context it is presented in and the purpose of the installation. Perhaps it is the role of the arts to ask these questions located at the fringes of information design by exploring these technologies as they are becoming accessible and ubiquitous.

References

GAVER, William; Beaver, Jake; Benford, Steve (2003), Ambiguity as a Resource for Design, Proceedings of CHI 2003, p. 233

ISHII, H., Dahley, A., and Wisneski, C. (1998a), Water Lamp and Pinwheels: Ambient projection of digital information into architectural space. Proceedings of $\mathrm{CHI}^{\prime} 98$ Companion (1998), ACM Press, p. 269-270

ISHII, H., Wisneski, C., Hiroshi, Dahley, A., Gorbet, M., Brave, S., Ullmer, B., Yarin, P., (1998b), Ambient Displays: Turning Architectural Space into an Interface between People and Digital Information, Published in the Proceedings of the First International Workshop on Cooperative Buildings (CoBuild '98), February 25-26, 1998, Springer

MANOVICH, Lev (2007), TATE Lecture, September 8th, 2007 manuscript available at http://www.manovich.com/TEXTS_07.HTM accessed April 27th 2008.

NISHIMURA, Yoshiaki (1999), Designing World-realm Experiences: The Absence of World Users at "Vision Plus 6" conference Vienna. Text available at http://www.sensorium.org/vp6/lecture/index.html accessed May 26th 2008

VLASTARAS, Dimitrios 2008, posting on January 10th 2008 at http://dimme.net/2008/01/10/webvisitors-blinker/ accessed May 27th 2008.

WEBER, Alexander (2007) posting on December 4th 2007 at http://tinkerlog.com/2007/12/04/arduino-xmas-hitcounter/ accessed May 27th 2008.

WEISER, Marc (1991), The computer for the 21st century. Scientific American, 1991. 165(3): p. 94-104

WEISER, Marc, Brown, John Seely (1995), "Designing Calm Technology", Xerox PARC. 21. 12 1995. Available at: http://www.ubiq.com/weiser/calmtech/calmtech.htm. Accessed on March 29th, 2008

WILSON, Stephen (2002), Information Arts: Intersections of art, science and technology," MIT Press, Cambridge, Massachusetts 